\title{
Duración de la psicosis no tratada: primeros pasos para la adopción de programas de intervención temprana de psicosis en el Perú
} Duration of untreated psychosis: first steps towards the adoption of early intervention programs of psychosis in Peru

Correspondencia

Rubén Valle

ruben_vr12@hotmail.com

Recibido: 04/08/2021

Aprobado: 09/09/2021

Citar como: Valle R. Duración de la psicosis no tratada: primeros pasos para la adopción de programas de intervención temprana de psicosis en el Perú. Acta Med Peru. 2021;38(3):237-8. doi: https://doi.org/10.35663/ amp.2021.383.2168

\section{Rubén Valle ${ }^{1}$}

Centro de Investigación en Epidemiología Clínica y Medicina Basada en Evidencias, Facultad de Medicina Humana, Universidad de San Martín de Porres, Lima, Perú

Señor editor:

La intervención temprana de psicosis es una estrategia eficaz y costo-efectiva para la recuperación clínica y funcional de personas que experimentan un primer episodio psicótico ${ }^{[1]}$. Uno de sus principales componentes es la detección temprana que busca identificar personas con tal sintomatología que no están siendo (adecuadamente) tratadas ${ }^{[2]}$. El periodo entre el inicio de los síntomas psicóticos y la primera toma de la medicación antipsicótica se denomina duración de la psicosis no tratada (Duration of untreated psychosis: DUP) ${ }^{[3]}$, y dura 63 semanas en países de altos ingresos y 125 semanas en países de bajos y mediados ingresos económicos ${ }^{[4]}$. Se ha postulado que durante esta etapa se produce un daño neurotóxico en las personas, por lo que los programas de intervención temprana de psicosis tienen como objetivo central reducir la DUP ${ }^{[3]}$.

Estudios con meta-análisis muestran los efectos de la DUP en la evolución clínica de los pacientes con un primer episodio de psicosis. La DUP prolongada se ha asociado a mayor sintomatología psicótica, bajo funcionamiento y pobre calidad de vida ${ }^{[3]}$. Además, la DUP corta se asocia con menos síntomas negativos, especialmente cuando la DUP es menor a 9 meses ${ }^{[5]}$. Aunque en general la DUP y el funcionamiento cognitivo no están relacionados, se observa una asociación significativa entre la DUP prolongada y la habilidad para planificar/resolver problemas ${ }^{[6]}$. Por otro lado, la DUP también se valora como un indicador de acceso a los servicios de salud mental por parte de los pacientes ${ }^{[7]}$. Estos encuentros resaltan la necesidad de implementar programas de intervención temprana de psicosis para detectar tempranamente los casos nuevos de esta patología.

Los programas de intervención temprana de psicosis son escasos en países de bajos y medianos ingresos económicos ${ }^{[4]}$. En América Latina solo algunos países han instalado centros donde se brindan este tipo de atención, pero no como una política nacional en salud mental ${ }^{[1]}$. El Perú aún no ha implementado un programa de tales características, por los que los pacientes que debutan con síntomas psicóticos reciben los mismos cuidados de salud que los pacientes en estadios crónicos ${ }^{[1]}$. No obstante, nosotros consideramos que hay dos estrategias de los programas de intervención temprana de psicosis que se podrían implementar en el actual sistema de salud mental del país, y que pueden informar sobre la frecuencia de presentación de casos nuevos de psicosis y del tiempo que demora recibir atención médica por esta patología en el Perú.

La primera estrategia es el registro diferenciado de "primer episodio de psicosis" en las historias clínicas y bases hospitalarias (distinto al de "paciente nuevo" que se usa en los centros de salud). Este registro 
permitiría constatar la atención de un primer caso de psicosis y medir los casos incidentes a nivel poblacional. La segunda es la evaluación (y registro en la historia clínica) de la DUP en la primera evaluación clínica. Esto podría brindar información pronóstica para su uso clínico; y su análisis sistematizado permitiría determinar el tiempo que demoran los pacientes con psicosis en acceder al tratamiento. De este modo, la aplicación de estas estrategias útiles y factibles de los programas de intervención temprana de psicosis podrían servir para mejorar la investigación, atención médica y salud pública en el área de psicosis en el Perú.

\section{REFERENCIAS}

1. Valle R. Revisión de los programas de intervención temprana de psicosis: propuesta de implementación en Perú. Rev Colomb Psiquiatr. 2020;49(3):178-86.
2. Marshall M, Rathbone J. Early intervention for psychosis. Schizophr Bull. 2011;37(6):1111-4.

3. Marshall $\mathrm{M}$, Lewis $\mathrm{S}$, Lockwood $\mathrm{A}$, Drake $\mathrm{R}$, Jones $\mathrm{P}$, Croudace $\mathrm{T}$. Association between duration of untreated psychosis and outcome in cohorts of first-episode patients: a systematic review. Arch Gen Psychiatry. 2005;62(9):975-83.

4. Large M, Farooq S, Nielssen $\mathrm{O}$, Slade T. Relationship between gross domestic product and duration of untreated psychosis in low- and middle-income countries. Br J Psychiatry. 2008;193(4):272-8.

5. Boonstra N, Klaassen R, Sytema S, Marshall M, De Haan L, Wunderink $\mathrm{L}$, et al. Duration of untreated psychosis and negative symptoms--a systematic review and meta-analysis of individual patient data. Schizophr Res. 2012;142(1-3):12-9.

6. Bora E, Yalincetin B, Akdede BB, Alptekin K. Duration of untreated psychosis and neurocognition in first-episode psychosis: A metaanalysis. Schizophr Res. 2018;193:3-10.

7. Brunet K, Birchwood M, Lester H, Thornhill K. Delays in mental health services and duration of untreated psychosis. Psychiatr Bull. 2007;31(11):408-10. 